\title{
Correlation between gastric volume and organs at risk dose in adjuvant radiotherapy for left breast cancer
}

\author{
Zahra Siavashpour ${ }^{1}{ }^{1}$, Mona Malekzadeh Moghani ${ }^{1}$, Neda Goharpey ${ }^{2}$, Hamid Reza Mirzai ${ }^{1}$, \\ Leila Kia Mohammadi², Farid Zayeri ${ }^{3}$ \\ ${ }^{1}$ Radiotherapy Oncology Department, Shohada Tajrish Hospital, Shahid Beheshti University of Medical Sciences, Tehran, Iran \\ ${ }^{2}$ Radiotherapy Oncology Department, Shohada Tajrish Hospital, Tehran, Iran \\ ${ }^{3}$ Proteomics Research Center and Biostatics Department, School of Allied Medical Sciences, Shahid Beheshti University of Medical Science, \\ Tehran, Iran
}

\begin{abstract}
Background: The role of the gastric volume on the dose-effect relationship for these organs has not been investigated. The aim of the study was to evaluate the correlation between gastric volume and dose-volume histogram (DVH) parameters of the heart, left lung and stomach during left breast cancer radiotherapy (RT).

Materials and methods: Ninety-nine left breast cancer patients who got adjuvant radiotherapy were included. Study was classified into two groups based on treatment field arrangements: 1) breast tangential fields only (T) and 2) breast tangential and supraclavicular fields (TS). Organs DVHs were extracted. Descriptive statistics, Pearson correlation, linear regression analyses, and receiver operating characteristic (ROC) analyses were performed.

Results: There is a direct but not significant correlation between the gastric volume and doses to the stomach and left lung. For a 100-cc increase in the gastric volume, the stomach maximum dose and the $V_{50}$ increased by 3 Gy and $4 \%$, respectively. For the left lung, $\mathrm{V}_{4}$ and $\mathrm{V}_{5}$ increased by $1 \%$ for TS cases. Considering ROC analysis results, one can make a decision for about $74 \%$ of patients due to their left lung DVH parameters, using gastric volume as a known input data. The correlation between gastric volume and heart dose was not significant.

Conclusions: The gastric volume of about $170 \mathrm{cc}$ or less can result in lower dose to the stomach and ipsilateral lung during left breast cancer radiotherapy, especially for TS cases. To reach this gastric volume threshold, patients should be fast for 2 hours before the procedure of $\mathrm{CT}$ simulation and treatment.
\end{abstract}

Key words: breast cancer; gastric volume; heart dose; lung dose

Rep Pract Oncol Radiother 2021;26(3):367-379

\section{Introduction}

In both developed and developing countries, breast cancer is still the most commonly diagnosed cancer among women, comprising $24.4 \%$ of all diagnosed malignancies among female cancer patients in Iran [1]. Various breast cancer man- agement strategies exist based on the clinical and pathological characteristics of patients and tumors. Typically, a multidisciplinary approach is used, in which radiotherapy (RT) accounts for about $87 \%$ of breast cancers [2].

The technical advancement from 3D conformal radiotherapy (3D-CRT) to intensity-modulated

Address for correspondence: Mona Malekzadeh Moghani, Department of RadioOncology, Shahid Beheshti University of Medical Sciences, Tehran, Iran, Shohada-e Tajrish Hospital, Department of RadioOncology, Shahid Beheshti University of Medical Sciences, Tehran, Iran, tel: +98912 309 8543; e-mail: m.malekzade20@gmail.com

This article is available in open access under Creative Common Attribution-Non-Commercial-No Derivatives 4.0 International (CC BY-NC-ND 4.0) license, allowing to download articles and share them with others as long as they credit the authors and the publisher, but without permission to change them in any way or use them commercially 
radiotherapy (IMRT) and volumetric modulated arc therapy (VMAT) has occurred for almost all treatment sites over the past two decades. IMRT provided superior dose conformity, especially for concave target volumes, and optimal dose sparing of adjacent normal tissues, but it might also increase the risk of radiation-induced secondary malignancies. Concerns about the secondary cancer of organs proximal to the fields, such as the contralateral breast, heart, and lung, have increased due to the higher low dose baths resulted from IMRT and VMAT [3]. Using the 3D-CRT technique, smaller volumes of normal tissues are potentially exposed to lower radiation dose than IMRT. However, due to its shorter planning time, there are still many cancer institutes that prefer the 3D-CRT technique for breast cancer radiotherapy, especially in high-loaded centers [4]. Furthermore, IMRT is still an expensive treatment procedure. In Iran, about 10,000 new cases of breast cancer are diagnosed every year which leads to overloaded public radiotherapy centers [5]. For all of these reasons, 3D-CRT is the first choice of breast cancer radiotherapy in Iranian centers. Moreover, conventional fractionation by $2 \mathrm{~Gy} /$ day and total dose of $50 \mathrm{~Gy}$, with/without boost dose to the tumor bed, has been prescribed as the standard of care [6].

Lungs, heart, and stomach are the considered organs at risk (OARs) in radiotherapy of the left breast. Exposure of these critical structures to radiation may cause early and late sequelae. Because of increasing the general education level and stronger prevention policies, it is hoped that cancers can be detected in early stages, so treatments can be started as soon as the diagnostic procedures are completed. Today, higher overall survival of breast cancer patients is expected. In this regard, late side effects of radio-therapeutic interventions and limiting the risk of secondary cancer deserve more attention.

Excess absolute risk (EAR) term has been defined to represent the risk of developing a solid secondary cancer after radiotherapy. This concept can be estimated from the DVH of treatment planning [7]. Several risk models have been proposed to predict secondary cancer but still there is no accurate prediction due to the lack of follow-up and epidemiological data [3].

The risk of secondary cancer for the ipsilateral lung was even estimated higher than for the other proximal organs; it receives a higher dose in order to achieve a higher tumor control. Generally, known side effects of left breast RT included secondary lung malignancy, pneumonitis, lung fibrosis, cardiovascular diseases, and potential gastric complications [8-10]. The dose-effect relationship was directly proportional to: the higher the dose, the greater the complication rate. Studies indicate that pneumonitis (chronic or acute) and fibrosis have a direct correlation with radiation doses to the lung $[11,12]$.

Heart complication after radiotherapy of breast cancer has previously been studied. Two studies confirmed the probability of the coronary complications, including revascularization and myocardial infarction, increased linearly with the mean dose to the heart $[13,14]$. The results of a cohort study proved that there was a significant dose-effect relationship between the probability of acute coronary events incidence and mean heart dose [15]. Cho et al. studied gastric complications after adjuvant radiotherapy for breast cancer. They concluded no chronic gastric complication was correlated with the radiation exposure during breast cancer irradiation [8]. Despite previous studies, the role of the gastric volume on the dose-effect relationship for the lung, heart, and stomach after the adjuvant left breast RT has not been investigated.

Therefore, to minimize the amount of low dose volume such as the known $\mathrm{V}_{4}$ and $\mathrm{V}_{5}\left(\mathrm{~V}_{\mathrm{x}}\right.$ referred to the volume receiving more than $\mathrm{x} G y)$, as well as the mean and maximum dose should be assessed for proximal OARs. This may reduce the risk of secondary cancer and other complications $[3,7]$.

This study aimed to evaluate the correlation between the gastric volume and the dose-volume histogram (DVH) parameters of the stomach, left lung, and heart during adjuvant radiotherapy for left breast cancer. This study also made an effort to propose a protocol for patients' gastric preparation before the procedure of $\mathrm{CT}$ simulation and treatment if any relationship between the DVH parameter and gastric volume was confirmed.

\section{Materials and methods}

\section{Study population}

Treatment planning data of 99 patients during two years from February 2018 to January 2020 were studied, retrospectively. In this study partici- 

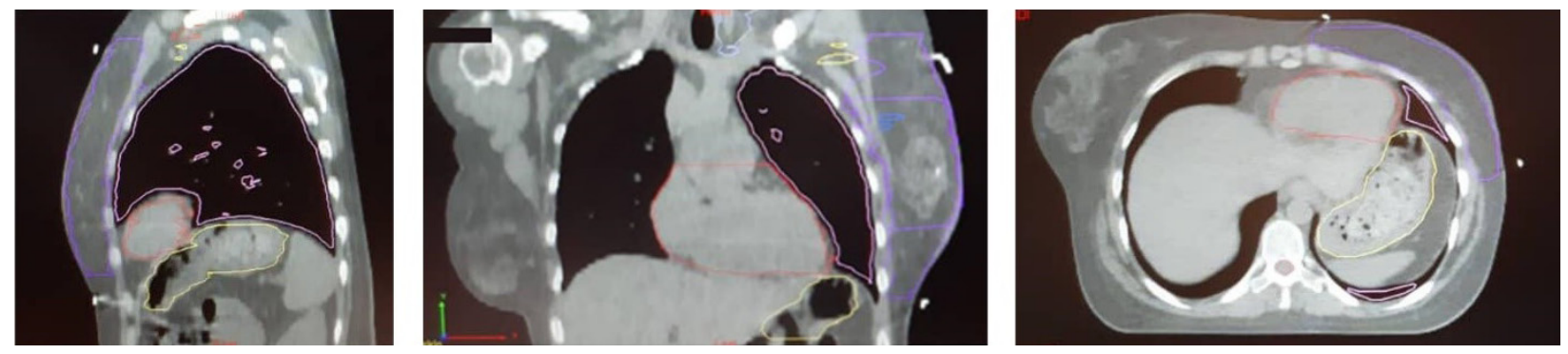

Figure 1. A sample of left breast case with her delineated organs

pants were patients treated by the 3D-CRT of the left breast cancer at Shohada-e Tarjish Hospital in Tehran, Iran. TNM and histological gradings were determined based on the National Comprehensive Cancer Network (NCCN) guidelines [16]. The patients were referred for postoperative irradiation based on their risk factors after mastectomy (MRM) or breast-conserving surgery (BCS) [17]. 83 of the included cases were BCS and the other 16 cases were MRM. The sampled population was classified into two groups according to the treatment field arrangements: 1) tangential (T) fields and 2) tangential/supraclavicular (TS) fields. All patients were set up in the supine position during CT simulations and radiotherapy treatments with the aid of a reproducible patient supporting system (AIO Breast; Orfit; Belgium). For none of the considered cases electron beam was used and all were treated by the $6 \mathrm{MV}$ photon beam of a Compact ${ }^{\mathrm{TM}}$ Linac (Elekta, Inc, Atlanta, USA). The treatment planning system was the ISOGray Solution (DOSIsoft Ed. 4; France). The considered treatment phase in this study for BCS cases was justphase I (through which the whole breast was irradiated) without inclusion of any boost irradiation. Posterior axillary field was used for all the selected TS cases.

\section{Contouring protocols}

Sampled population involved patients with CT scan images covering the whole gastric volume. Left breast, chest wall, as well as auxiliary and supraclavicular lymph nodes were contoured based on the RTOG guideline [18]. Heart contour included the pulmonary artery and extended in the inferior direction to the apex [19]. The gastric volume contoured began at the gastro-esophageal junction (GEJ) and ended at the pyloric canal opened to the duodenum. The cardia, fungus, body or corpus, antrum, and pylorus were included in the stomach structure $[20,21]$. All delineated structures were reviewed by an expertise for consistency in contouring. A sample of a left breast case with its delineated organs has been illustrated in Figure 1.

\section{Data collection}

Treatment plans were evaluated in accordance with the national and international clinical protocols at the time of planning [22-26]. All of the dose constraints are according to QUANTEC. For example, for the lung: $\mathrm{V}_{20}<30 \%$, for the heart: mean $\mathrm{Gy}, \mathrm{V}_{25}<10 \%$. All of the included patients were treated by the conventional dose-fractionation of 50 Gy in 25 fractions. The DVH parameters of the stomach, left lung, and heart were extracted from the approved treatment plans and used in the following statistical analyses. These parameters included the understudied organs $V_{x}$ for different $\mathrm{x}$ Gy ranging from $4 \mathrm{~Gy}$ (as the low dose) to 50 Gy (as the prescription dose), their average/mean dose, and their maximum dose as presented in Table 1.

\section{Statistical analysis}

Descriptive statistics was used to describe included patients and treatment characteristics. Pearson correlation and linear regression analysis were used to investigate the relationship between the DVH endpoints of OAR (stomach; left lung; heart) and the gastric volume using a statistical significance level of 0.05 ( $\mathrm{p}$-value $<0.05$ ). Receiver operating characteristic (ROC) analysis was also used to validate the results calculated by the two statistical models. The area under the curve (AUC) metric evaluated the classification performance of the ROC model and assisted in determining the threshold of the gastric volume, when a strong correlation with the DVH endpoint was confirmed 
Table 1. Descriptive statistics for patients' dose-volume histogram (DVH) parameters

\begin{tabular}{|c|c|c|c|c|c|c|c|c|c|c|c|c|c|}
\hline Organ & \multicolumn{2}{|c|}{ Volume (cc) } & \multicolumn{9}{|c|}{ Volume (\%) } & \multicolumn{2}{|c|}{ Dose [Gy] } \\
\hline \multirow{5}{*}{ Gastric } & & & & & & $V_{45}$ & $V_{50}$ & $V_{55}$ & & & & $D_{\operatorname{Max}}$ & $\mathrm{D}_{\text {Mean }}$ \\
\hline & Min & 69.00 & & & & 0.00 & 0.00 & 0.00 & & & & 0.00 & 0.64 \\
\hline & Max & 984.00 & & & & 21.50 & 5.50 & 0.00 & & & & 51.97 & 16.30 \\
\hline & Mean & 258.87 & & & & 1.15 & 0.11 & 0.00 & & & & 31.55 & 3.48 \\
\hline & SD & 180.95 & & & & 3.27 & 0.68 & 0.00 & & & & 18.65 & 3.13 \\
\hline \multirow{5}{*}{$\begin{array}{l}\text { Left } \\
\text { lung }\end{array}$} & & & $V_{4}$ & $V_{5}$ & $\mathrm{~V}_{8}$ & $V_{10}$ & $V_{11}$ & $\mathrm{~V}_{12.5}$ & $V_{16}$ & $V_{20}$ & $V_{30}$ & & $D_{\text {Mean }}$ \\
\hline & Min & 441.00 & 13.40 & 10.30 & 6.00 & 4.90 & 4.40 & 4.00 & 3.20 & 2.50 & 1.50 & & 2.60 \\
\hline & Max & 1371.00 & 68.90 & 62.80 & 50.10 & 46.40 & 44.80 & 43.20 & 40.50 & 38.30 & 34.30 & & 19.90 \\
\hline & Mean & 915.36 & 44.49 & 39.80 & 31.74 & 28.95 & 27.93 & 26.73 & 24.79 & 23.12 & 20.29 & & 12.88 \\
\hline & SD & 183.76 & 13.16 & 12.27 & 10.28 & 9.56 & 9.30 & 9.00 & 8.53 & 8.10 & 7.45 & & 4.03 \\
\hline \multirow{5}{*}{ Heart } & & & & & $\mathrm{V}_{8}$ & $V_{10}$ & $V_{16}$ & $\mathrm{~V}_{20}$ & $V_{25}$ & $V_{30}$ & & & $\mathrm{D}_{\text {Mean }}$ \\
\hline & Min & 250.00 & & & 3.05 & 2.67 & 2.06 & 1.74 & 1.53 & 1.23 & & & 1.60 \\
\hline & Max & 1046.00 & & & 50.60 & 35.30 & 32.70 & 31.60 & 30.10 & 28.74 & & & 11.20 \\
\hline & Mean & 539.65 & & & 15.63 & 14.71 & 12.81 & 12.07 & 11.97 & 10.72 & & & 8.58 \\
\hline & SD & 141.68 & & & 7.92 & 7.01 & 6.62 & 6.42 & 8.14 & 6.03 & & & 11.00 \\
\hline
\end{tabular}

$V_{x}$ - the percent volume of organ receiving a dose of $X$ Gy or more; $D_{\text {Max }}$ - maximum dose to the OARs; $D_{\text {Mean }}$ - mean dose of OARs; OAR - organ at risk

[27]. All of the statistical analyses were carried out in the IBM SPSS Statistics (Version 23, IBM Crop.) Software.

\section{Results}

\section{Descriptive statistics}

Table 1 describes the statistics of the physical and dosimetric parameters of the three normal organs (stomach; left lung; heart) after the 3D-CRT of the left breast. The total sample size is 99: 51 cases were classified into the T group, and 48 cases into the TS group. Distribution of the patient's age ranged from 28 to 76, with the average age of 49 and standard deviation of 13. Categories of T1, T2, and T3 for staging of the primary tumor's size accounted for $46 \%, 19 \%$, and $65 \%$, respectively. Categories of N0, $\mathrm{N} 1, \mathrm{~N} 2$, and $\mathrm{N} 3$ for staging of the spread to nearby lymph nodes accounted for 65\%, 19\%, 11\%, and $5 \%$, respectively. Histologic grading of G1, G2, and G3 for classification of cancer cells' growth and spread rates accounted for $21 \%, 65 \%$, and $14 \%$, respectively, of the total sample population. To be candidate for TS treatment patients had to have some indications; reported positive lymph nodes, categorized in T3 or T4 stages, not enough axillary lymph nodes were dissected or the result of sentinel biopsy was not convincing, and sometimes had medial lesions.

\section{Correlation tests}

Table 2 summarizes the Pearson's correlation and the linear regression results for potential correlation between the gastric volume and the dosimetric parameters (shown in Tab. 1) for the stomach, left lung, and heart. Separate results were shown in the table for the T and TS cases in each statistical test. Figures 2-4 show the linear regression models for the relationships between the gastric volume and the $\mathrm{V}_{4}$ and $\mathrm{V}_{5}$ of the left lung, and $\mathrm{D}_{\mathrm{Max}}$ of the stomach, respectively. For all of them the p-value was less than 0.05 and statistically significant, as shown in Table 2. Separate plots of the regression model were shown in these figures for the $\mathrm{T}$ and $\mathrm{TS}$ groups for each corresponding dosimetric parameter.

Based on the obtained Pearson's correlation coefficients (R) in Table 2, there are direct but very weak to moderate correlations between the gastric volume and the dose received by the stomach, heart and left lung for these cases. Non-zero and positive regression coefficients mean that by any increase in gastric volume, the related DVH of the stomach, left lung, and heart will increase. However, this relationship was statistically significant just for $\mathrm{V}_{4}$ and $\mathrm{V}_{5}$ of left lung in TS cases ( $\mathrm{p}$-value $<0.05$ ).

Moreover, Figures 2-4 were plotted to make an easier illustration and discern the correlations between the DVHs parameters and gastric volume. These figures and their trendlines slope give an in- 
Table 2. Correlation between gastric volume and the stomach, left lung, and heart dose-volume histogram (DVH) parameters. T and TS cases are considered separately

\begin{tabular}{|c|c|c|c|c|c|c|c|c|c|c|c|c|c|}
\hline Organ & \multicolumn{11}{|c|}{ Volume (\%) } & \multicolumn{2}{|c|}{ Dose [Gy] } \\
\hline \multirow{7}{*}{ Gastric } & & & & & & $\mathrm{V}_{45}$ & $\mathrm{~V}_{50}$ & & & & & $\mathrm{D}_{\operatorname{Max}}$ & $\mathrm{D}_{\text {Mean }}$ \\
\hline & $\mathrm{R}$ & $\mathrm{T}$ & & & & 0.31 & 0.49 & & & & & 0.31 & 0.27 \\
\hline & & TS & & & & 0.12 & 0.03 & & & & & 0.27 & 0.09 \\
\hline & R.C. & $\mathrm{T}$ & & & & $0.01^{*}$ & 0.04 & & & & & $0.03^{* *}$ & 0.00 \\
\hline & & TS & & & & 0.00 & 0.00 & & & & & 0.03 & 0.00 \\
\hline & P-value & $\mathrm{T}$ & & & & 0.02 & 0.00 & & & & & 0.03 & 0.06 \\
\hline & & TS & & & & 0.41 & 0.83 & & & & & 0.07 & 0.56 \\
\hline \multirow{7}{*}{$\begin{array}{l}\text { Left } \\
\text { lung }\end{array}$} & & & $V_{4}$ & $V_{5}$ & $V_{8}$ & $V_{10}$ & $V_{11}$ & $\mathrm{~V}_{12.5}$ & $V_{16}$ & $V_{20}$ & $V_{30}$ & & $\mathrm{D}_{\text {Mean }}$ \\
\hline & $\mathrm{R}$ & $\mathrm{T}$ & 0.09 & 0.09 & 0.10 & 0.11 & 0.11 & 0.11 & 0.12 & 0.11 & 0.11 & & 0.12 \\
\hline & & TS & 0.33 & 0.31 & 0.22 & 0.22 & 0.21 & 0.20 & 0.18 & 0.16 & 0.14 & & 0.17 \\
\hline & R.C. & $T$ & 0.00 & 0.00 & 0.00 & 0.00 & 0.00 & 0.00 & 0.00 & 0.00 & 0.00 & & 0.00 \\
\hline & & TS & 0.01 & 0.01 & 0.01 & 0.01 & 0.01 & 0.01 & 0.01 & 0.01 & 0.00 & & 0.00 \\
\hline & P-value & $\mathrm{T}$ & 0.53 & 0.52 & 0.47 & 0.44 & 0.43 & 0.42 & 0.42 & 0.43 & 0.42 & & 0.42 \\
\hline & & TS & 0.02 & 0.03 & 0.14 & 0.13 & 0.16 & 0.18 & 0.22 & 0.29 & 0.36 & & 0.24 \\
\hline \multirow{7}{*}{ Heart } & & & & & $V_{8}$ & $V_{10}$ & $V_{16}$ & $\mathrm{~V}_{20}$ & $V_{25}$ & $V_{30}$ & & & $\mathrm{D}_{\text {Mean }}$ \\
\hline & $\mathrm{R}$ & $\mathrm{T}$ & & & 0.03 & 0.03 & 0.04 & 0.04 & 0.29 & 0.01 & & & 0.05 \\
\hline & & TS & & & 0.05 & 0.08 & 0.08 & 0.09 & 0.09 & 0.09 & & & -0.08 \\
\hline & R.C. & $T$ & & & 0.00 & 0.00 & 0.00 & 0.00 & 0.01 & 0.00 & & & 0.00 \\
\hline & & TS & & & 0.00 & 0.00 & 0.00 & 0.00 & 0.00 & 0.00 & & & 0.01 \\
\hline & P-value & $\mathrm{T}$ & & & 0.83 & 0.81 & 0.78 & 0.80 & 0.04 & 0.94 & & & 0.72 \\
\hline & & TS & & & 0.76 & 0.60 & 0.59 & 0.56 & 0.53 & 0.54 & & & 0.59 \\
\hline
\end{tabular}

$V_{x}$ - the percent volume of organ receiving a dose of $X$ Gy or more; $D_{\text {Max }}$ - maximum dose to the delineated OAR; $D_{\text {Mean }}-$ radiation doses averaged across delineated OAR; T — cases treated with tangential fields; TS — cases treated with tangential/supraclavicular fields; R - Pearson's Correlation; RC — regression coefficient; *by every $1 \mathrm{cc}$ increase in gastric volume its $\mathrm{V}_{45}$ will increase by $0.01 \%$ and so on for the other RC obtained for OARs' $\mathrm{V}_{x ;}{ }^{* *}$ by every $1 \mathrm{cc}^{\prime}$ increase in gastric volume its $D_{\text {Max }}$ will increase by 0.03 Gy and so on for the other R.C. obtained for OARs' $D_{\text {Max }}$ and $D_{\text {Mean }}$

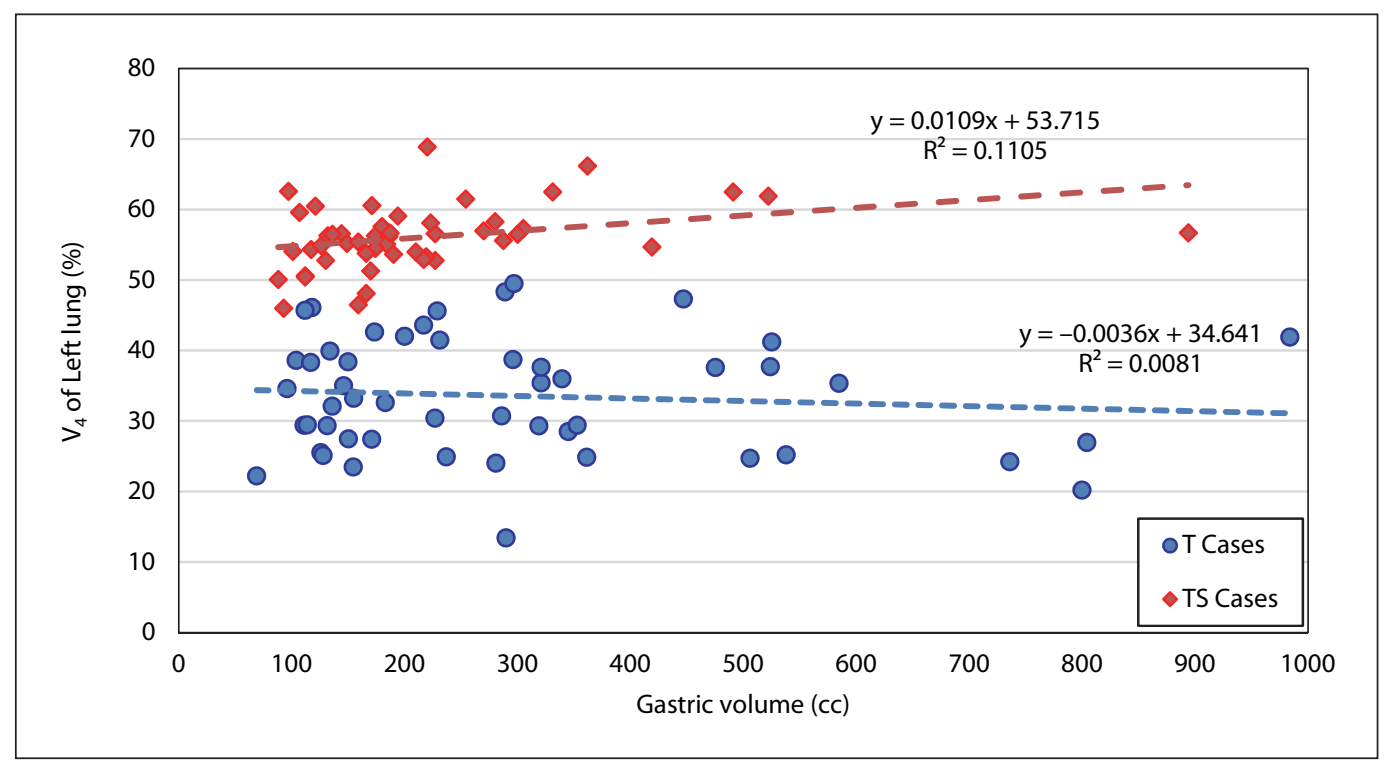

Figure 2. Correlation between gastric volume and $\mathrm{V}_{4}$ of left lung 


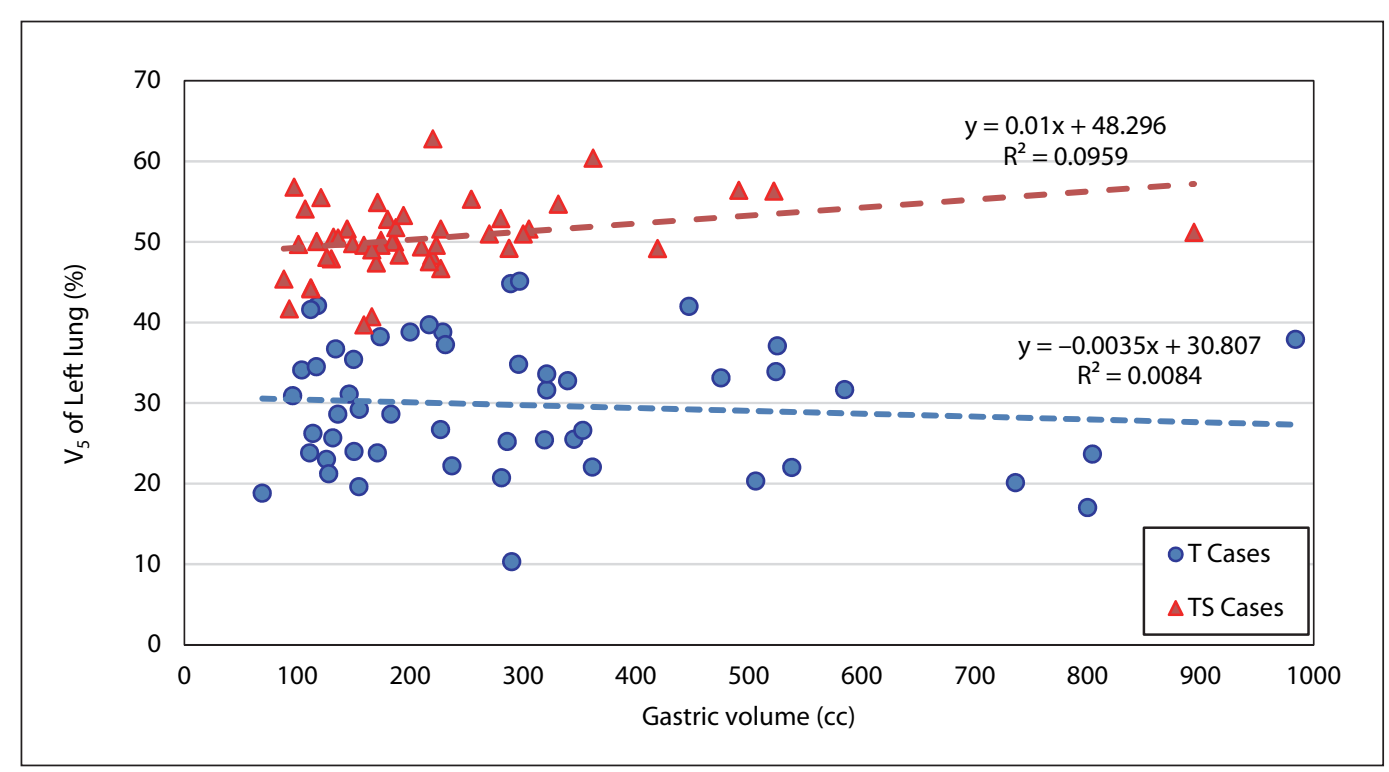

Figure 3. Correlation between gastric volume and $V_{5}$ of left lung

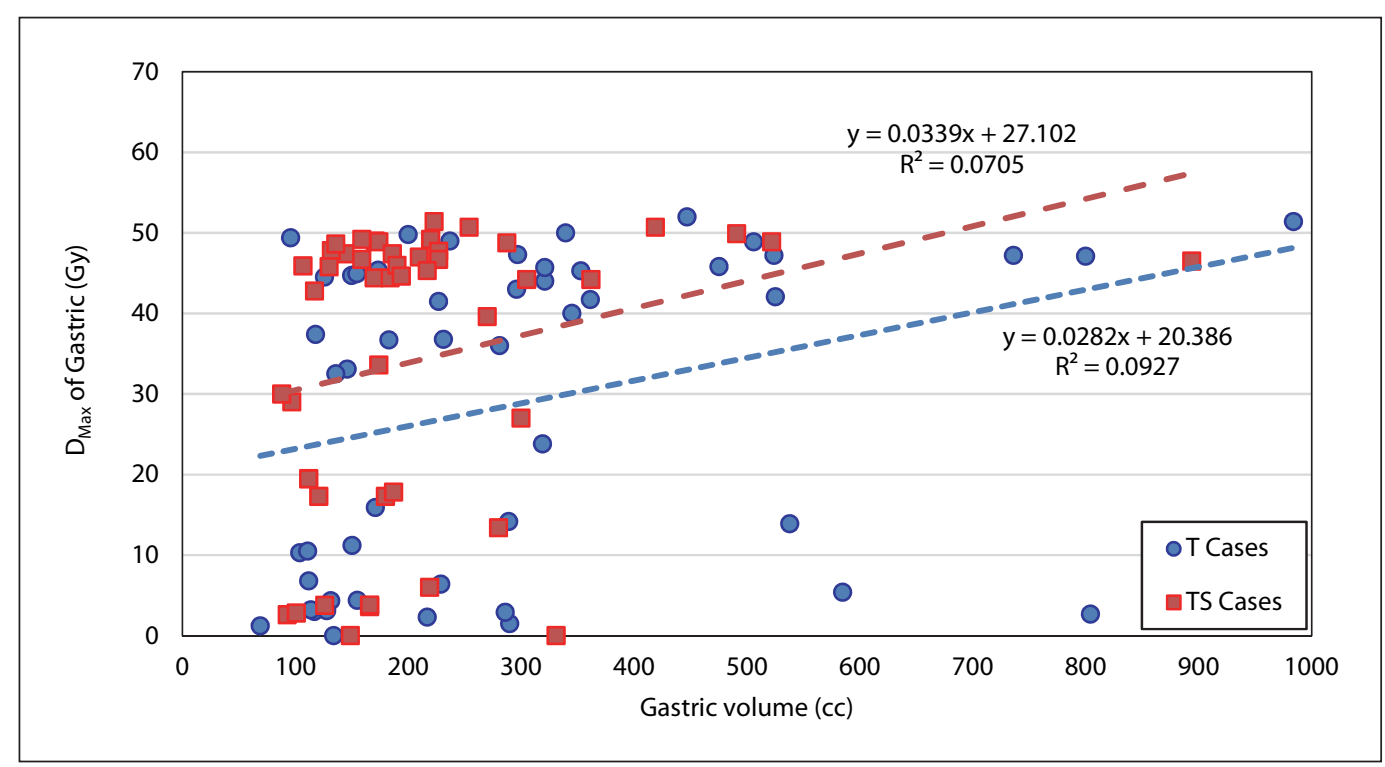

Figure 4. Correlation between gastric volume and $D_{\operatorname{Max}}$ of gastric

sight into the nature of the actual data, especially for the most significant results obtained from Table 2 (i.e. $V_{4}, V_{5}$ of the lung and $D_{\operatorname{Max}}$ of the stomach).

There is again a positive but weak to moderate association between maximum dose of the gastric volume and its volume for both $\mathrm{T}$ and $\mathrm{TS}$ groups. In each 100-cc increase in the gastric volume, its maximum dose would probably increase by $3 \mathrm{~Gy}$, based on the obtained regression coefficients in Table 2.

There is no significant observed relationship between the gastric volume and heart dose during the left breast RT (Tab. 2). However, just for $\mathrm{V}_{25}$ of the heart in cases with tangential fields, there was a positive but not strong correlation with a reportable R.C. that can be explained as before (i.e. by increasing $100 \mathrm{cc}$ of gastric volume, $\mathrm{V}_{25}$ of the heart would probably increase by $1 \%$ ).

\section{ROC analysis}

Using the descriptive analysis of DVH parameters (Mean \pm SD) in addition to dose constraints suggested in the protocols mentioned earlier 
Table 3. Receiver operating characteristic (ROC) curve characteristics for dose-volume histogram (DVH) parameters

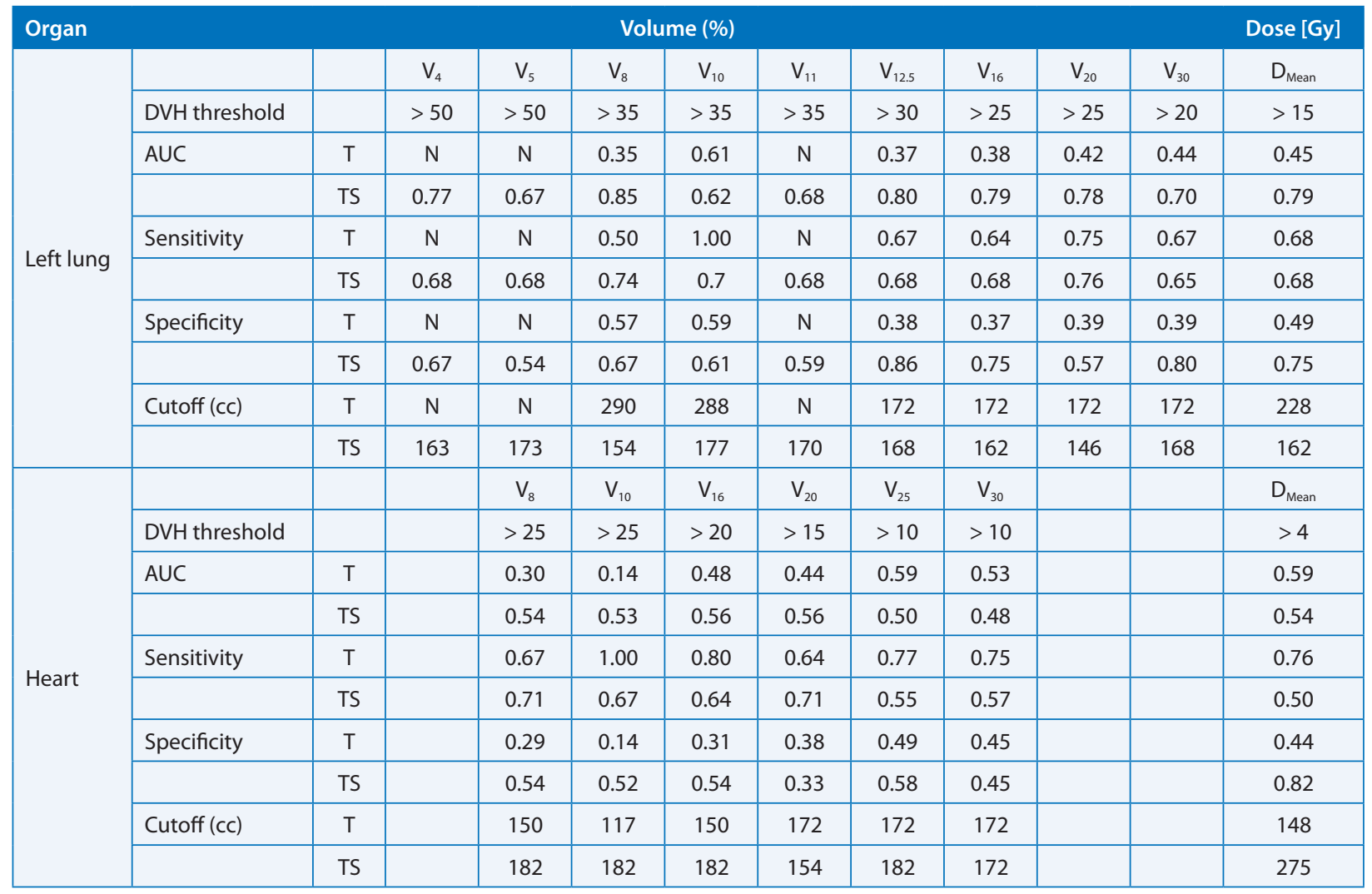

$V_{x}$ : - the percent volume of organ receiving a dose of X Gy or more; $D_{\text {Mean }}$ - mean dose of OARs; AUC — area under the curve; $T$ - cases treated with tangential fields; TS - cases treated with tangential/supraclavicular fields; $\mathrm{N}$ - non reportable due to the low sample size

(QUANTEC, EMAMI, RTOG 1005, and NSABP B-51), DVH thresholds were selected for the ROC analysis (Tab. 3 and Fig. 5-7).

The ROC analyses between the gastric volume and its $\mathrm{V}_{45}, \mathrm{~V}_{50}$, and $\mathrm{V}_{55}$ parameters resulted in no predictive ability of gastric volume for these DVH parameters. However, the resulted AUCs of analysis between gastric volume and its maximum dose were 0.77 (sensitivity $=0.80$ and specificity $=0.61$ ) and 0.65 (sensitivity $=0.61$ and specificity $=0.64$ ) for $\mathrm{T}$ and $\mathrm{TS}$ groups, respectively (Fig. 5). The cut-off point of gastric volume for $\mathrm{T}$ cases was about $230 \mathrm{cc}$ and for TS cases was about $185 \mathrm{cc}$. These results were obtained after classification of $D_{\text {Max }}$ into two binary groups of $0: D_{\text {Max }} \leq 45$ Gy and 1: $\mathrm{D}_{\text {Max }}>45 \mathrm{~Gy}$, the tested state was 1 . This analysis was also repeated for average dose of the stomach. For T cases no meaningful results were obtained after binary classification of the dose $\left(0: D_{\text {Mean }} \leq 10\right.$ Gy and 1: $\left.D_{\text {Mean }}>10 \mathrm{~Gy}\right)$. For the TS group and with the gastric volume cut-off point of about $220 \mathrm{cc}$, AUC was 0.59 with sensitivity and specificity of 0.67 and 0.69 , respectively. Other reportable ROC curves for the left lung and heart DVH parameters are illustrated in Figures 6 and 7 and the analyses detailed results are presented in Table 3 .

\section{Discussion}

In different radiotherapy departments, based on their available image guidance facilities, such as electronic portal imaging device (EPID), cone-beam CT (CBCT) or even using MR-linac, several universal and/or local protocols are followed for patients' pre-simulation and pre-treatment preparation to reduce the radiation dose received by OARs and improve the adaptive treatment reproducibility [28]. However, there is no written preparation protocol for breast cancer irradiation regarding the gastric volume and, because of that, the high standard deviation of $181 \mathrm{cc}$ (ranged from about $69 \mathrm{cc}$ to 984 cc) for the gastric volume was achieved in Table 1.

In adjuvant $\mathrm{RT}$ of left-sided breast cancer, treating regional lymph node led to a remarkable increase in heart and lung doses [29]. Based on the recent systematic review by Anzar et al. $M L D_{\text {ipsi }}$ in 

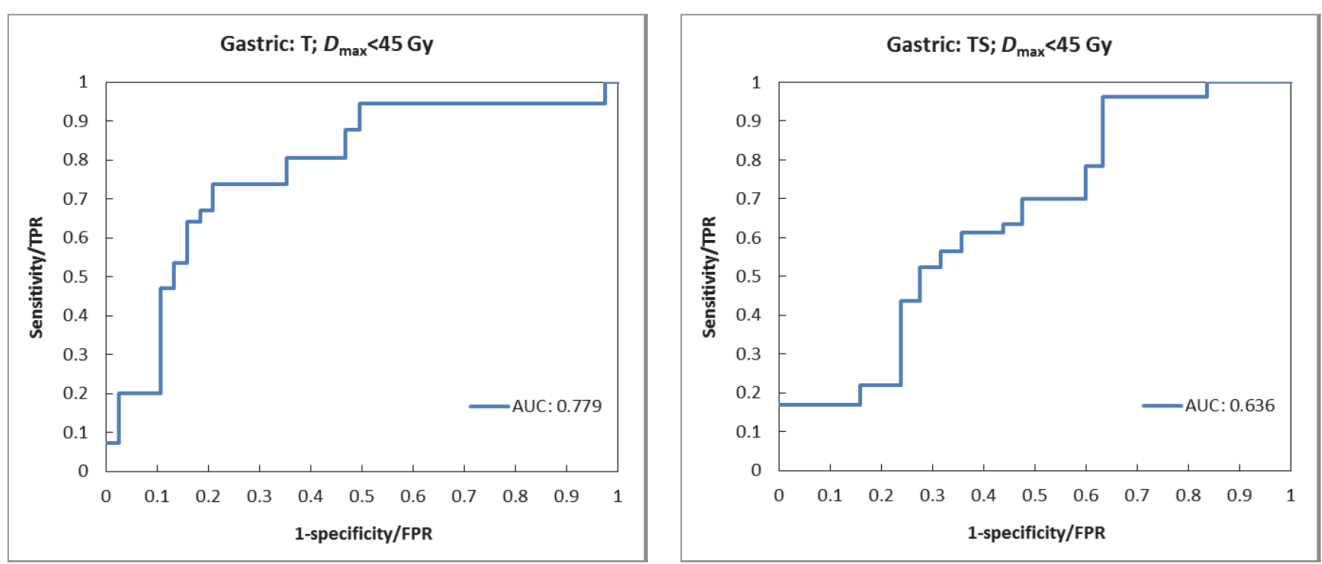

Figure 5. ROC analysis results of gastric volume predictability for its maximum dose. $D_{\text {Max }}$ was classified into two binary groups; 0: Gastric $D_{\operatorname{Max}} \leq 45$ Gy and 1: Gastric $D_{\operatorname{Max}}>45$ Gy and the tested state was 1. Left picture is for $T$ (cases treated with tangential fields), and the right one for TS (cases treated with tangential/supraclavicular fields) groups
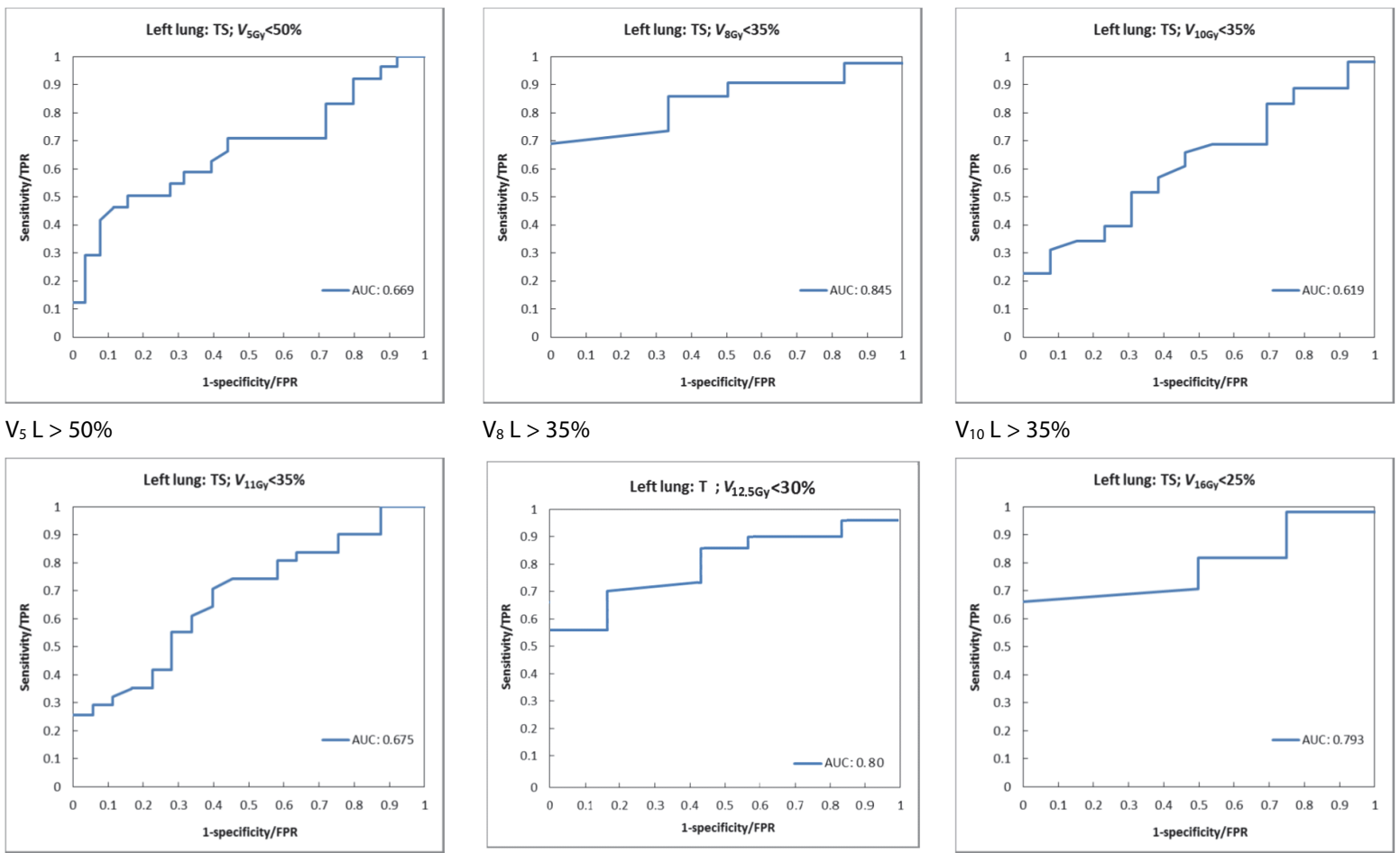

\section{$V_{8} L>35 \%$}

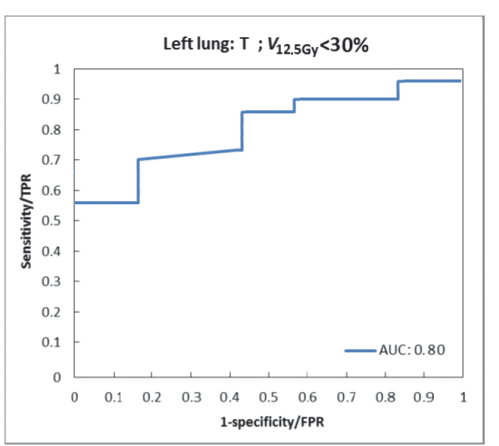

\section{$V_{10} L>35 \%$}
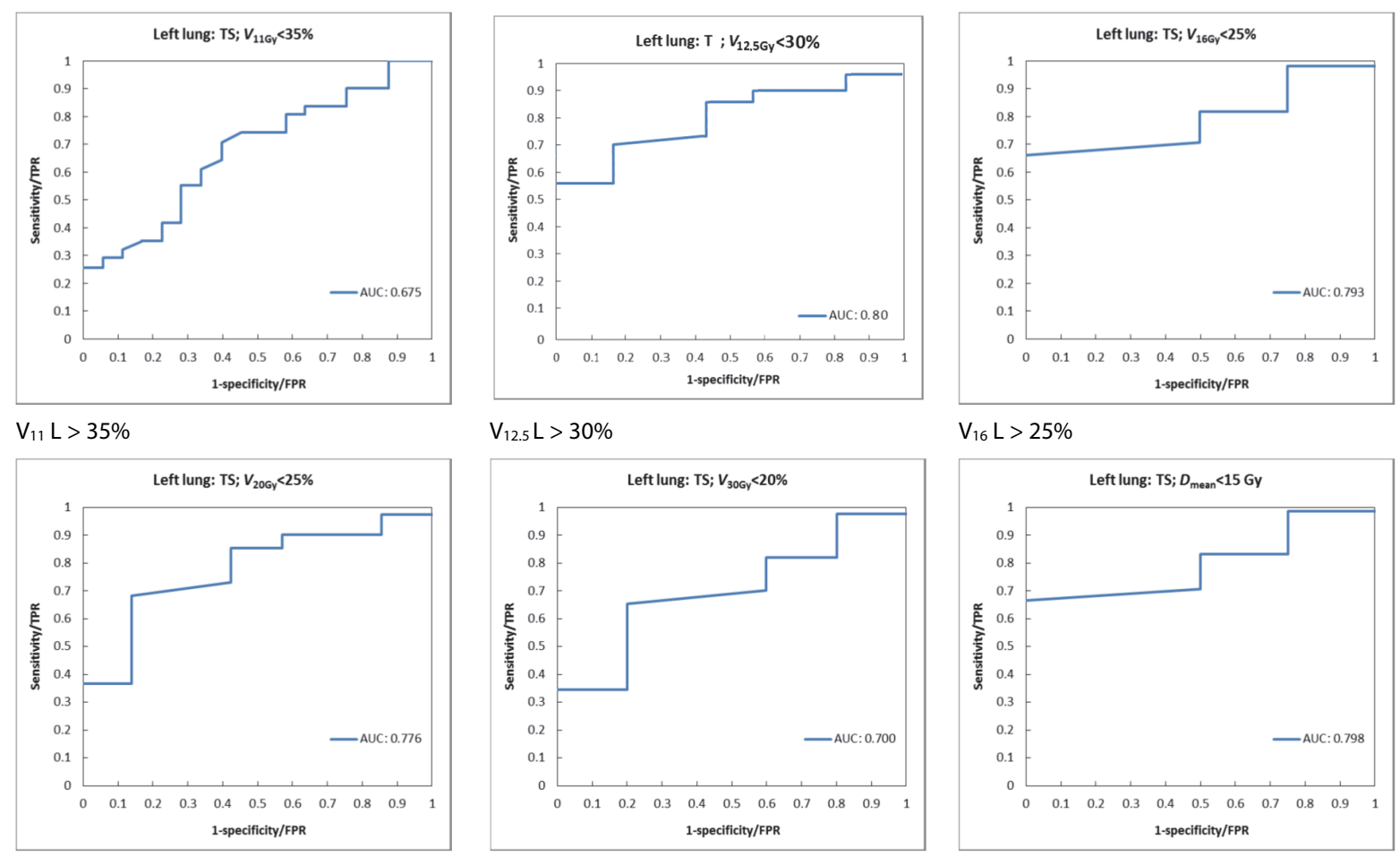

Figure 6. ROC analysis results of gastric volume predictability for left lung DVH parameters ( $\mathrm{L}-$ left lung). T — cases treated with tangential fields; TS — cases treated with tangential/supraclavicular fields 

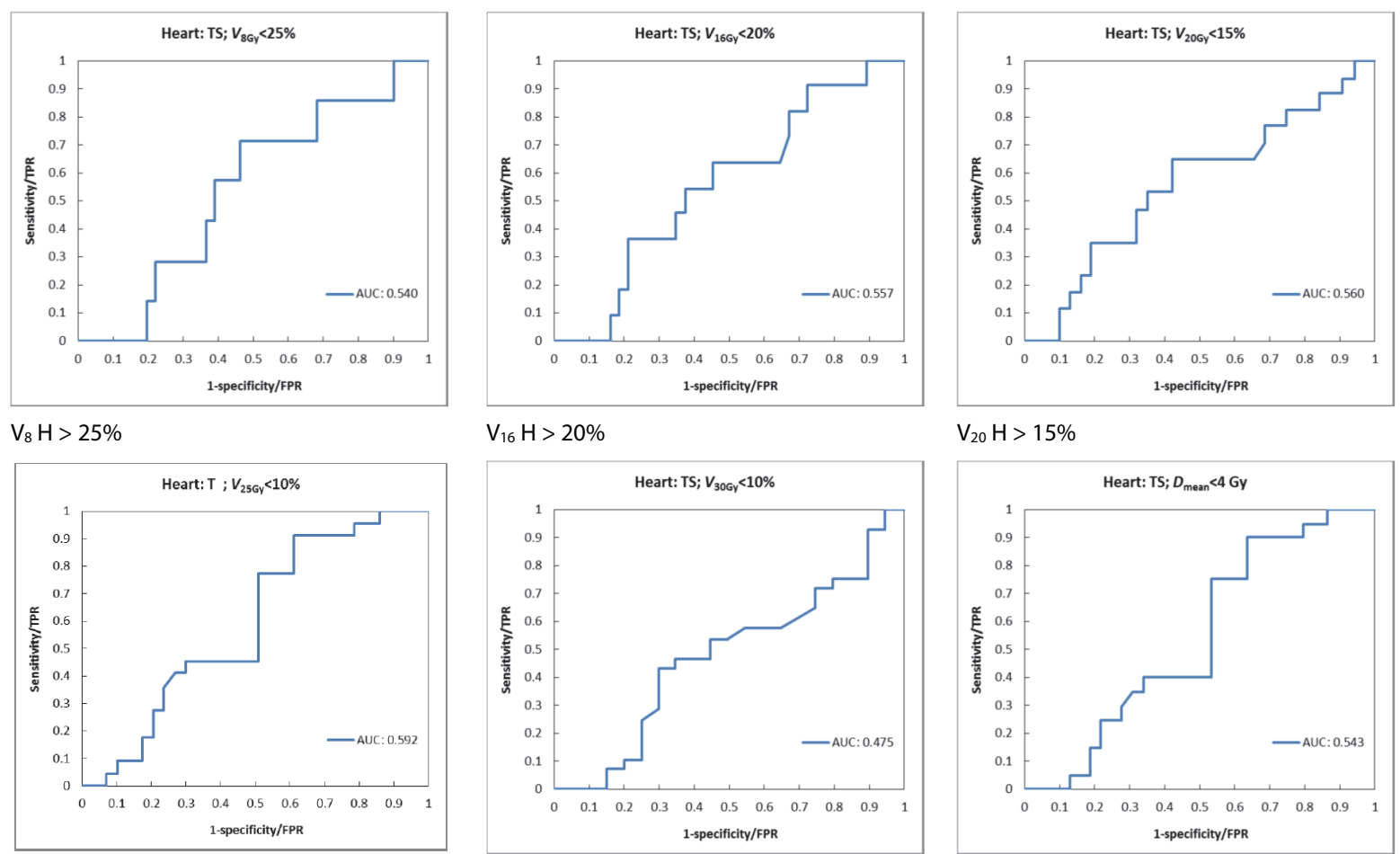

$\mathrm{V}_{25} \mathrm{H}>10 \%$

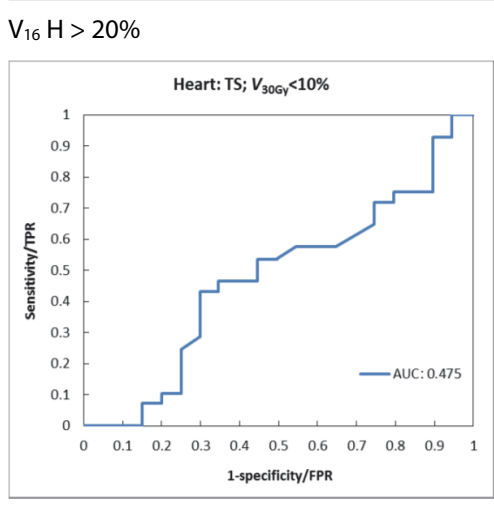

$\mathrm{V}_{30} \mathrm{H}>10$

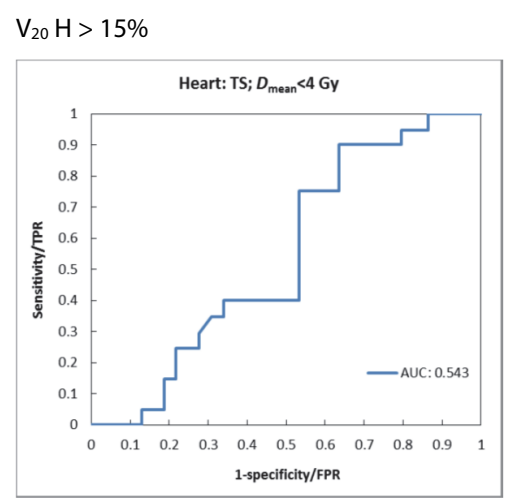

$D_{\text {Mean }} \mathrm{H}>4 \mathrm{~Gy}$

Figure 7. Receiver operating characteristic $(\mathrm{ROC})$ analysis results of gastric volume predictability for heart dose-volume histogram (DVH) parameters. $\mathrm{H}$ - heart; $\mathrm{T}$ - cases treated with tangential fields; $\mathrm{TS}$ - cases treated with tangential/ /supraclavicular fields

breast radiotherapy with no breathing adaption in a supine position, it was 8.4 Gy for whole breast or/and chest-wall RT and 11.2 Gy when the axilla or/and supraclavicular fossa was irradiated [12]. That's why considered cases were classified into two groups of T and TS in the current study.

The mean \pm SD of the left lung dose obtained $12.9 \pm 4.0$ Gy which was close to the data reported by Anzar MC et al. from their meta-analysis of conformal radiotherapy with tangent fields $(10.9 \pm 0.8 \mathrm{~Gy})$ and IMRT (11.7 $\pm 1.1 \mathrm{~Gy}$ ) [12]. Referring to the $\mathrm{D}_{\text {Mean }}$ definition, any fluctuation in the lung volume can influence the resultant mean dose. Mean \pm SD of the left lung volume was $915.4 \pm 183.8 \mathrm{cc}$ for the cases being studied. Not following any breathing protocols during simulation can add more lung volume variations beside the intrinsic variation between different patients' anatomies. Therefore, high standard deviation could be due to the absence of any breathing protocols during patients' CT simulation.

Considering the recent publication by Cho WK et al., a five-year follow-up of breast cancer patients indicated that adjuvant RT is not a risk factor in late gastric cancer [8]. Despite the fact that the stomach is considered as a radioresistant organ (maximum tissue tolerance dose with $5 \%$ rate of gastric complication in 5 years (TD 5/5) is 50 Gy based on Emami B. et al.), gastric ulceration risk would be $16 \%$ if it receives more than $50 \mathrm{~Gy}$ dose as reported by Brick IB et al. [26, 30]. For T cases, the regression coefficient (R.C.) was 0.04 (Tab. 2), which means that by increasing gastric volume in every $100 \mathrm{cc}$ its $\mathrm{V}_{50}$ may increase by $4 \%$. Therefore, fuller stomach increases the risk of gastric ulceration during left breast irradiation.

Based on Table 2, dependency of the ipsilateral lung volume that would receive low dose on the gastric volume is higher for TS cases than T ones. The apex of lung is irradiated to a higher dose by the tangential/supraclavicular RT fields and a higher correlation coefficient for TS was obtained compared to the $\mathrm{T}$ group referring to their Pearson coefficients (e.g. 0.09 for T vs. 0.33 for the TS cases' $\mathrm{V}_{4}$ ). Using this direct correlation, the dose given to the lung can be managed better by controlling the gastric volume. For example, by considering regression coefficients of TS cases, one can conclude that an increase of $100 \mathrm{cc}$ in gastric volume 
may significantly raise $\mathrm{V}_{4}$ and $\mathrm{V}_{5}$ of the left lung by 1 percent $(100 \mathrm{cc} \times 0.01=1)$ with $\mathrm{p}$-value of 0.02 and 0.03 , respectively. As mentioned, this correlation is much stronger in treatment of lymph node positive cases (TS) than the negative ones (T). The other DVH parameters of the left lung had a direct but moderate relationship with the gastric volume by approximately the same regression coefficients. However, the obtained associations were not strong (based on the calculated Pearson correlation coefficients) and not significant (based on the P-values) for this study population. Based on previous studies, such as Zhao $\mathrm{H}$ et al. and Drachman CB et al. review articles, radiation-induced secondary cancer is a concern that needs attention, especially in the irradiated organ volumes where the received dose from the primary fields exceeds 2.5 Gy [31, 32]. Furthermore, due to an individual patient data meta-analysis of 75 randomized trials, every $1 \mathrm{~Gy}$ increase in the mean dose of the lung during the radiotherapy of breast cancer, the risk of lung cancer increases by $11 \%$ after a decade from radiotherapy $[12,33]$. However, there is no significant correlation ( $p$-value $>0.05$ ) between the mean dose of the left lung and the gastric volume in the current study. This randomized trial was performed by Taylor et al. and they also reported that the average of ipsilateral lung dose in modern breast radiotherapy was 9.0 Gy and the average of whole-lung dose was about 5.0 Gy. They also calculated the excess rate ratio (ERR) per Gy of whole-lung dose for incidence of lung cancer for two smoking and non-smoking groups. Based on their obtained ERR, the risk of mortality from radiation induced lung cancer is much higher in the smoking (from 9.4\% to 13.8\%) than nonsmoking group (from $0.5 \%$ to $0.8 \%$ ). If the whole lung dose becomes higher, the excess risk will also be higher [33].

Based on previous studies, even a small heart dose can lead to cardiac disease as shown by the relative increase in this complication per gray [13]. There is a negative correlation [but weak and not significant ( $\mathrm{p}$-value $>0.05$ )] between the mean dose of the heart and the gastric volume for the current TS cases (by R.C. $\approx-0.01$; by every increase of $100 \mathrm{cc}$ in the gastric volume the heart mean dose may decrease by 1 Gy). Darby et al. reported the linear correlation coefficient between the rate of acute coronary events and heart mean dose $(7.4 \%$ per 1 Gy) after radiotherapy of the left breast [14].
Van Den Bogaard et al. studied the relationship between acute coronary disease and the mean dose of the heart and indicated that its cumulative incidence increased by $16.5 \%$ per 1 Gy mean heart dose 9 years after this treatment [15]. Beaton et al. also assessed the cardiovascular disease (CVD) of women after 10 years of their left breast RT and checked if QUANTEC guidelines violation had any relationship with dying from this disease [9]. They concluded that the risk of death from CVD after 10 years of left breast RT is very low if the heart mean dose had been kept less than 3.3 Gy. However, weak correlation was detected between the heart dose and gastric volume in the current study.

AUC was approximately 0.77 for ROC analysis between the gastric volume and its maximum dose, which means that the gastric volume can classify about $77 \%$ of patients and specify whether their $\mathrm{D}_{\text {max }}$ of the stomach is more than $45 \mathrm{~Gy}$ or not (Fig. 5). Also, the sensitivity and specificity of 0.80 and 0.61 show that by possessing $185 \mathrm{cc}$ as a cut-off point for gastric volume, the $\mathrm{D}_{\max }$ of the stomach in $80 \%$ of patients can be predicted to be more than 45 Gy and in $61 \%$ of them to be less than 45 Gy.

The column $\mathrm{V}_{4}$ of the left lung for TS cases in Table 3 can be interpreted based on the estimated indices. For this column, the AUC of 0.77 tells us that by considering the gastric volume one can truly classify about $77 \%$ of patients based on whether their left lung $\mathrm{V}_{4}$ is more than $50 \%$ or not. The obtained sensitivity of 0.68 for TS cases with gastric volume cut-off of about $163 \mathrm{cc}$ indicates that using this cut-off point, one can truly predict about $68 \%$ of patients' left lung $\mathrm{V}_{4}$ parameters to be more than $50 \%$. One the other hand, its specificity of 0.67 also indicates that by using this cut-off point it is predictable for about $67 \%$ of patients' $\mathrm{V}_{4}$ of the stomach to be less than $50 \%$.

Similar to $\mathrm{V}_{4}$, using AUCs calculated for the other left lung DVH parameters of TS cases (Tab. 3), by knowing the gastric volume one can truly classify about $67 \%, 85 \%, 62 \%, 68 \%, 80 \%, 79 \%, 78 \%, 70 \%$, and $79 \%$ of patients based on whether their left lung $\mathrm{V}_{5}, \mathrm{~V}_{8}, \mathrm{~V}_{10}, \mathrm{~V}_{11}, \mathrm{~V}_{12.5}, \mathrm{~V}_{16}, \mathrm{~V}_{20}, \mathrm{~V}_{30}$, and $\mathrm{D}_{\text {Mean }}$ is more than $50 \%, 35 \%, 35 \%, 35 \%, 30 \%, 25 \%, 25 \%, 20 \%$, and 15 Gy or not, respectively. Eventually, by using gastric volume data one may probably make a good prediction for about $75 \%$ (i.e. the average of all left lung DVH parameters' AUC) of these patients' left lung DVH parameters. 
AUCs of $\mathrm{T}$ cases were almost less than 0.50, which means that by considering the gastric volume as a known data, DVH parameters of the ipsilateral lung can be foreseen for less than 50\% of patients. However, just for $\mathrm{V}_{10}$ of the left lung in T cases, AUC obtained was 0.61, which means that by managing the gastric volume (with a cut-off point of about $288 \mathrm{cc}$ ) just for $61 \%$ of patients, one can truly predict whether left lung $\mathrm{V}_{10}$ is more than $35 \%$ or not.

AUC resulted from ROC analysis of TS cases, the $\mathrm{V}_{8}, \mathrm{~V}_{10}, \mathrm{~V}_{16}$, and $\mathrm{V}_{20}$ parameters of the heart with thresholds of $25 \%, 25 \%, 20 \%$, and $15 \%$ were about $0.54,0.53,0.56$, and 0.56 , respectively. For example, the column $\mathrm{V}_{10}$ of the heart with $25 \mathrm{~Gy}$ threshold can be explained so that just 53\% of patients can be truly classified based on whether their heart $\mathrm{V}_{10}$ is less than 25 Gy or not. The sensitivity of 0.67 with $182 \mathrm{cc}$ cut-off point for the gastric volume shows that for $67 \%$ of patients, $V_{10}$ of the heart can be predicted to be less than $25 \%$. Nevertheless, the specificity of 0.52 shows that by using this cut-off point for $52 \%$ of patients, $\mathrm{V}_{10}$ of the heart can be predicted to be more than 25\%, which is not reliable predictor data. Therefore, gastric volume is not a good predictor of the heart dose neither for TS nor for T cases. However, as a suggestion for future study, the heart's left descending artery (LAD) and left ventricle (LV) could also be delineated and assessed by having patients' CT scans acquired with a contrast agent to see if there is any correlation between their dose and gastric volume.

The average of all gastric volume cut-off points for the studied DVH parameters of the left lung and heart were $161 \pm 12 \mathrm{cc}$ and $176 \pm 11 \mathrm{cc}$, respectively. Therefore, it can be concluded that the gastric volume of about $170 \mathrm{cc}$ can result in an optimal situation for the stomach, left lung, and heart dose reduction during left breast cancer radiotherapy, especially with tangential plus supraclavicular fields. Based on the Hunt et al. study, 2 hours fasting can lead to an empty stomach [34]. There are also some previous studies that followed 2 hours fasting to reach a semi-empty stomach for radiotherapy purpose [35]. Therefore, considering about 2 hours fasting before CT simulation and every radiotherapy fraction can help patients reach this gastric volume and subsequent OARs' dose. In addition, by this gastric volume the control of the risk of secondary cancer for the left lung would probably decrease, especially for TS cases, due to the reduction of its low dose.

Low statistical population can be named as the most limitation of current cross-sectional study. The non-uniformity of some ROC curves in Figures 5-7 with non-rounded shape was apparently due to the inadequate sample size in its related subgroup. The authors suggest a future prospective study on the DVH parameters of each mentioned OARs and follow up the related radiotherapy sequelae. Patients can be classified based not only on their $\mathrm{T}$ or TS prescribed treatment status but also based on their body surface area (BSA) which could lead to some bias to the current study results.

Eventually, to be able to suggest some recommendations as a patient preparation protocol for left breast cancer adjuvant radiotherapy, more multicentric randomized trial and meta-analysis studies are required. Image guided radiotherapy with cone beam CT or even MRI can give more reliable data about the exact position of soft tissues, like the stomach, during radiotherapy and its impact on the anatomical shift of its surrounding organs.

\section{Conclusions}

In this retrospective study, the effect of gastric volume on dose received by the stomach, heart, and left lung was evaluated during adjuvant radiotherapy of left breast cancer patients. Due to the obtained correlations, decreasing the gastric volume would reduce the ipsilateral lung mean dose. It also decreases the volumes that reach the low dose during left breast irradiation. This dose reduction may reduce the risk of patients' secondary lung cancer during the following decades of RT. The correlations between the gastric volume and heart DVH parameters was found non-significant. Maximum dose to the stomach is associated with its volume during RT. Any non-invasive interference that could reduce these OARs' dose would be appreciated during left breast radiotherapy.

\section{Conflict of interest}

There is no conflict of interest.

\section{Funding}

None declared. 


\section{References}

1. Farhood B, Geraily G, Alizadeh A. Incidence and Mortality of Various Cancers in Iran and Compare to Other Countries: A Review Article. Iran J Public Health. 2018; 47(3): 309-316, indexed in Pubmed: 29845017.

2. Joiner M, Van der Kogel A. Basic Clinical Radiobiology. 5th ed. Taylor \& Francis Group, New York 2019.

3. Zhang Q, Liu J, Ao N, et al. Secondary cancer risk after radiation therapy for breast cancer with different radiotherapy techniques. Sci Rep. 2020; 10(1): 1220, doi: 10.1038/ s41598-020-58134-z, indexed in Pubmed: 31988348.

4. Rastogi K, Sharma S, Gupta S, et al. Dosimetric comparison of IMRT versus 3DCRT for post-mastectomy chest wall irradiation. Radiat Oncol J. 2018;36(1): 71-78, doi: 10.3857/ roj.2017.00381, indexed in Pubmed: 29621872.

5. Nafissi N, Khayamzadeh M, Zeinali Z, et al. Epidemiology and Histopathology of Breast Cancer in Iran versus Other Middle Eastern Countries. Mid East J Cancer. 2018; 9(3): 243-251.

6. Smith BD, Bellon JR, Blitzblau R, et al. Radiation therapy for the whole breast: Executive summary of an American Society for Radiation Oncology (ASTRO) evidence-based guideline. Pract Radiat Oncol. 2018; 8(3): 145-152, doi: 10.1016/j.prro.2018.01.012, indexed in Pubmed: 29545124.

7. Abo-Madyan Y, Aziz MH, Aly MM, et al. Second cancer risk after 3D-CRT, IMRT and VMAT for breast cancer. Radiother Oncol. 2014; 110(3): 471-476, doi: 10.1016/j. radonc.2013.12.002, indexed in Pubmed: 24444525.

8. Cho WK, Choi DHo, Park W, et al. Gastric Complications after Adjuvant Radiotherapy for Breast Cancer. J Breast Cancer. 2019; 22(3): 464-471, doi: 10.4048/jbc.2019.22. e42, indexed in Pubmed: 31598345.

9. Beaton L, Bergman A, Nichol A, et al. Cardiac death after breast radiotherapy and the QUANTEC cardiac guidelines. Clin Transl Radiat Oncol. 2019; 19: 39-45, doi: 10.1016/j. ctro.2019.08.001, indexed in Pubmed: 31485490.

10. Lee D, Dinniwell R, Lee G. A Retrospective Analysis of Lung Volume and Cardiac Dose in Left-Sided Whole Breast Radiotherapy. J Med Imaging Radiat Sci. 2016; 47(3S): S10-S14, doi: 10.1016/j.jmir.2016.04.008, indexed in Pubmed: 31047480.

11. Omarini C, Thanopoulou E, Johnston SRD. Pneumonitis and pulmonary fibrosis associated with breast cancer treatments. Breast Cancer Res Treat. 2014; 146(2): 245-258, doi: 10.1007/s10549-014-3016-5, indexed in Pubmed: 24929676.

12. Aznar MC, Duane FK, Darby SC, et al. Exposure of the lungs in breast cancer radiotherapy: A systematic review of lung doses published 2010-2015. Radiother Oncol. 2018; 126(1): 148-154, doi: 10.1016/j.radonc.2017.11.022, indexed in Pubmed: 29246585.

13. Piroth MD, Baumann R, Budach W, et al. Heart toxicity from breast cancer radiotherapy : Current findings, assessment, and prevention. Strahlenther Onkol. 2019; 195(1): 1-12, doi: 10.1007/s00066-018-1378-z, indexed in Pubmed: 30310926.

14. Duane FK, McGale P, Brønnum D, et al. Risk of ischemic heart disease in women after radiotherapy for breast cancer. N Engl J Med. 2013; 368(11): 987-998, doi: 10.1056/ NEJMoa1209825, indexed in Pubmed: 23484825.
15. van den Bogaard VAB, Ta BDP, van der Schaaf A, et al. Validation and Modification of a Prediction Model for Acute Cardiac Events in Patients With Breast Cancer Treated With Radiotherapy Based on Three-Dimensional Dose Distributions to Cardiac Substructures. J Clin Oncol. 2017; 35(11): 1171-1178, doi: 10.1200/JCO.2016.69.8480, indexed in Pubmed: 28095159.

16. Breast cancer. National Comprehensive Cancer Network (NCCN Guidelines ${ }^{\circledR}$ ); 2020. . https://www.nccn.org/professionals/physician_gls/pdf/breast.pdf (April 2020).

17. Halperin EC, Wazer DE, Perez CA, Brady LW. Perez \& Brady's Principles and Practice of Radiation Oncology. 7th ed. Wolters Kluwer, New York 2019.

18. Breast Cancer Atlas for Radiation Therapy Planning: Consensus Definitions. RTOG Foundations INC. https://www. srobf.cz/downloads/cilove-objemy/breastcanceratlas. pdf (Jan 2018).

19. RTOG 1106: Atlases for Organs at Risk (OARs) in Thoracic Radiation Therapy. RTOG FoundationINC. https://www. rtog.org/LinkClick.aspx?fileticket=VyMTDbz25wY\%3d\& tabid=361 (March 2020).

20. Upper Abdominal Normal Organ Contouring Guidelines RTOG FOUNDATION INC; 2013. https://www.rtog. org/LinkClick.aspx?fileticket=dgwtfz553_g\%3d\&tab id=387 (March 2020).

21. Cefaro GA, Genovesi D, Perez CA. Delineating Organs at Risk in Radiation Therapy. Springer, London 2013: 33-34.

22. A Phase III Trial Of Accelerated Whole Breast Irradiation With Hypofractionation Plus Concurrent Boost Versus Standard Whole Breast Irradiation Plus Sequential Boost For Early-Stage Breast Cancer NRG Oncology 2014. https:// www.rtog.org/ClinicalTrials/ProtocolTable/StudyDetails. aspx?action=openFile\&FileID=9366 (March 2020).

23. A Randomized Phase III Clinical Trial Evaluating PostMastectomy Chestwall and Regional Nodal XRT and Post-Lumpectomy Regional Nodal XRT in Patients with Positive Axillary Nodes Before Neoadjuvant Chemotherapy Who Convert to Pathologically Negative Axillary Nodes After Neoadjuvant Chemotherapy. NRG ONCOLOGY; 2016. https://www.nrgoncology.org/ClinicalTrials/Protocol/nsabp-b-51-rtog-1304?filter=nsabp-b-51rtog-1304 (March 2020).

24. Gagliardi G, Constine LS, Moiseenko V, et al. Radiation dose-volume effects in the heart. Int J Radiat Oncol Biol Phys. 2010; 76(3 Suppl): S77-S85, doi: 10.1016/j. ijrobp.2009.04.093, indexed in Pubmed: 20171522.

25. Marks LB, Bentzen SM, Deasy JO, et al. Radiation dose-volume effects in the lung. Int J Radiat Oncol Biol Phys. 2010; 76(3 Suppl): S70-S76, doi: 10.1016/j.ijrobp.2009.06.091, indexed in Pubmed: 20171521.

26. Emami B, Lyman J, Brown A, et al. Tolerance of normal tissue to therapeutic irradiation. Int J Radiat Oncol Biol Phys. 1991;21(1): 109-122, doi: 10.1016/0360-3016(91)90171-y, indexed in Pubmed: 2032882.

27. Hajian-Tilaki K. Receiver Operating Characteristic (ROC) Curve Analysis for Medical Diagnostic Test Evaluation. Caspian J Intern Med. 2013; 4(2): 627-635, indexed in Pubmed: 24009950.

28. Zhang S, Yu YH, Zhang Y, et al. Radiotherapy in muscleinvasive bladder cancer: the latest research progress and clinical application. Am J Cancer Res. 2015; 5(2): 854-868, indexed in Pubmed: 25973322. 
29. Finazzi T, Nguyen VT, Zimmermann F, et al. Impact of patient and treatment characteristics on heart and lung dose in adjuvant radiotherapy for left-sided breast cancer. Radiat Oncol. 2019; 14(1): 153, doi: 10.1186/s13014-0191364-3, indexed in Pubmed: 31462267.

30. Brick IB. Effects of million volt irradiation on the gastrointestinal tract. AMA Arch Intern Med. 1955; 96(1): 26-31, doi: 10.1001/archinte.1955.04430010040004, indexed in Pubmed: 14387317.

31. Zhao H, He M, Cheng G, et al. A comparative dosimetric study of left sided breast cancer after breast-conserving surgery treated with VMAT and IMRT. Radiat Oncol. 2015; 10: 231, doi: 10.1186/s13014-015-0531-4, indexed in Pubmed: 26577189.

32. Dracham CB, Shankar A, Madan R. Radiation induced secondary malignancies: a review article. Radiat Oncol J.
2018; 36(2): 85-94, doi: 10.3857/roj.2018.00290, indexed in Pubmed: 29983028.

33. Taylor C, Correa C, Duane FK, et al. Early Breast Cancer Trialists' Collaborative Group. Estimating the Risks of Breast Cancer Radiotherapy: Evidence From Modern Radiation Doses to the Lungs and Heart and From Previous Randomized Trials. J Clin Oncol. 2017; 35(15): 1641-1649, doi: 10.1200/ JCO.2016.72.0722, indexed in Pubmed: 28319436.

34. Hunt JN, Spurrell WR. The pattern of emptying of the human stomach. J Physiol. 1951; 113(2-3): 157168, doi: 10.1113/jphysiol.1951.sp004562, indexed in Pubmed: 14832765.

35. Tuglu O, Gultekin M, Ozyigit G, et al. Effect of Gastric Filling on Radiation Therapy in Patients with Gastrointesinal Cancer. Int J Radiat Oncol Biol Phys. 2018; 102(3): e492, doi: 10.1016/j.ijrobp.2018.07.1399. 